\title{
Multi-objectives and Simulation Models for Develop Production Line in Automobiles Manufacturing System
}

\author{
Razman Bin Tahar, Ali A. J Adham \\ University Malaysia Pahang, Gambang, Kuantan Pahang, Malaysia
}

\begin{abstract}
Automotive manufacturing is complex and includes the coordination of design in the manufacturing system. The manufacturing approaches over the past few years, disassembly have been a key issue, and it seems that simulation models are usually tailored to address a narrow set of industrial issues. This paper describes the development of the production line in the automobile manufacturing system through design, operation, and maintenance, based on multi-objectives of Algorithm and Simulation Model called MOA-SM. The both models are being developed at three different levels: the production line in the body and assembly shop, supply chain, and the production plan. The optimization tries to involve more objectives to solve the issues in manufacturing system. A solution that may optimize one performance measure may deteriorate since other performance solutions are difficult. The resulting algorithms are comparable to the simulation and multi-object in terms of success rate, assembly times, peak forces, moments, and have assembly times superior to those of a benchmark blind search algorithm.
\end{abstract}

Keywords: assembly line, automotive manufacturing, supply chain, simulation model, multi-objective, production line

\section{Introduction}

Vehicles successively pass through three different shops (body, painting and assembly), and each of these shops has specific constraints and perturbations that locally modify the pre-defined sequence of vehicles (Kapadia, 1998). Assembly lines consist of a set of stations that produce one or many products. Each product must go through each station in the same order. In order for an assembly line to function properly the operations in the stations, it must be balanced with respect to cycle time (Zoroufi, 2004). The analysis evaluated the cost structure for conventional vehicle manufacturing, retailing and assigned shares of the manufacturer's suggested retail price (MSRP) to various cost contributors (Deogratias, 2007).

In an increasingly competitive world, the multi-objectives and simulation models has become a very powerful tool for the planning, design, and control of manufacturing systems to facilitate current and future, training, experimentation, and testing of interoperability of software (Angelisa, 2003). Manufacturing simulation is one of the most powerful analysis tools available to those responsible for the design and operation of manufacturing systems, and the model can be reconfigured for many situations in the automotive assembly. The automobile manufacturing is carefully reexamining operating policies and procedures, typical manufacturing competitive priorities or strategies are low cost, delivery, flexibility and quality (Chen, 2009).

Razman Bin Tahar, Ph.D., professor, Faculty of Technology Management, University Malaysia Pahang.

Ali A. J Adham, Ph.D. candidate, Faculty of Technology Management, University Malaysia Pahang.

Correspondence concerning this article should be Ali A. J Adham, Faculty of Technology Management, University Malaysia Pahang, Gambang, Pahang, 26300, Malaysia. E-mail: aliadham12@yahoo.com. 
Generic simulation identifies common model input and output data interfaces that could be standardized for particular modeling level and simulation case studies (Benny, 2008). The company has applied simulation into many of the decision making processes, and it is intrinsically a demanding and resource intensive task involving at least two major activities: model building and experimentation (Razmin \& Ali, 2009). A multi-objective programming (MOP) model was applied to the feed formulation process with the objectives of minimizing nutrient variance and minimizing ration cost, and the level of mobility that is socially optimal is the amount and mix of production activity that consumers would choose in an efficient market that reflects the principles of efficient pricing, consumer options and optimal planning.

This paper focuses on development of vehicle manufacturing system design, operation, and maintenance based on multi-objectives algorithm and simulation. The first goal is to improve the products line, and the second goal enhancing supply chain is to lower the product time, the third goal is to develop production plan. The process involves a number of operations, which require assembling together thousands of fabricated and purchased components, subassemblies, and systems. For supporting the manufacturers, researchers and standards, organizations using MATLAB reality simulation addition the overall goal to virtual manufacturing environment process to provide interoperability tests environment. The sequence of the work presented here are:

- Identifying facilities, systems, operations, parts, and processes to develop the production line in the automotive manufacturing;

- Developing the production plan by using multi-objective and simulation models;

- Performing conformance and acceptance testing using simulations and multi-objective to create the specified range of inputs for a delivered system or process;

- Evaluating effectiveness of new interface standards and protocol to meet manufacturing industry needs.

Finally, a result shows that the company could explore new operating procedures, organizational structures, etc., without disrupting ongoing operations and the time can be controlled: It can be compressed, expanded, etc., allowing the speeding or slowing down of the product plan.

\section{Theoretical Background}

\section{Manufacturing Strategy}

Manufacturing strategy has been defined as the pattern of decisions that, over time, enables a business unit to achieve a desired manufacturing structure, infrastructure and set of specific capabilities.

Many older vehicles had separate underlying stiffening structures and bodies, the body housing the passengers. This design requires more materials and results in a heavier car, thus raising costs. The automotive production process consists of three major sections: the body shop, the paint shop, and the trim assembly shop. Other sections are the power train assembly (consisting of the engine, gearbox, clutch, and transmission), and the press shop if body parts are stamped at the plant. There is also a final testing process where vehicles are checked for water tightness and a stationary road test (Deogratias, 2007). Some parts are usually assembled into the body before it is merged with the power system; the process is called "body drop", since it is the body that is usually lowered onto the chassis/power system (Dalvi, 2009). These most trim assembly operations are carried out manually. Typically, there is a worker or two on either side of the line at a station. There is sufficient space on which to work, equipment and tools specific to the station, and there are racked or bins on which the parts are stored for assembly. This is where a variety of configuration options can be made by the customer. The options on the car are that it can be either two or four doors. A two-door car will require a different body side panel from a four-door car. 
An assembly line is a special case of the product layout. Adopting a product layout makes sense when the batch size of a given product or part is large relative to the number of different products or parts produced. Assembly lines refer to progressive assembly operations linked by some material handling devices. Virtually, any product that has multiple parts and is produced in large volume is produced on assembly lines to some degree (Liu, Da, \& Han, 1999).

\section{Multi-objective Model}

Multi-objective model can be found in various fields: product and process design, finance, aircraft design, automobile design, or wherever optimal decisions need to be taken in the presence of trade-offs between two or more conflicting objectives (Javadian, 2007). Maximizing profit and minimizing the cost of a product, maximizing performance and minimizing fuel consumption of a vehicle, and minimizing weight while maximizing the strength of a particular component are examples of multi-objective optimization problems. A general multi-objective optimization problem can be defined as follows (Zitzler, 1999):

$$
\operatorname{Max} n / \operatorname{Min} Y_{i}=f_{i}(x)=\left(f_{1}(x), f_{2}(x) \ldots f_{n}(x)\right)
$$

Subject to:

$$
\begin{aligned}
& x=\left(x_{1}, x_{2}, \ldots, x_{n}\right) \in X \\
& y=\left(y_{1}, y_{2} \ldots . . y_{n}\right) \in Y
\end{aligned}
$$

where, $x$ is the decision vector, $y$ is the objective vector; $X$ is the parameter space, and $Y$ is the objective space.

\section{Simulation Model}

Simulation is one of the most powerful analysis tools available to those responsible for the design and/or operation of complex processes or systems (Fritz, 1997). It is heavily based upon computer science, mathematics, probability theory and statistics, yet the process of simulation modeling and experimentation remains very much an intuitive art. Simulation is a very general and somewhat ill-defined subject.

Simulation can be defined as the process of designing a model of a real system and conducting experiments with this model for the purpose of understanding the behavior of the system and/or evaluating various strategies for the operation of the system. Simulation includes both the construction of the model and the experimental use of the model for studying a problem.

\section{The Proposed Method}

\section{Multi-objective Formula}

The automotive component manufacturing multi-objectives model with the goal of developing a procedure to improve labor utilization arid thereby cuts the cost of labor. This is further proof that the above stated objective was successfully satisfied. Multiple-objective programming (MOP) model was applied to the feed formulation process with the objectives of minimizing nutrient variance and minimizing ration cost, the level of mobility which is socially optimal is the amount and mix of transport activity that consumers would choose in an efficient market that reflects the principles of efficient pricing, consumer options and optimal planning.

If a multi-objective problem is well formed, there should not be a single solution that simultaneously minimizes each objective to its fullest. In each case, we are looking for a solution for which each objective has been optimized to the extent that if we try to optimize it any further, the other objective(s) will suffer as a result. Finding such a solution, and quantifying how much better this solution is compared to other such as solutions (there will generally be many) are the goal when setting up and solving a multi-objective optimization problem. 
Figure 1 shows the product stage of producing car. The logistics and manufacturing strategies should be aligned with the supply chain strategy in order to build the necessary capabilities to address customer priorities.

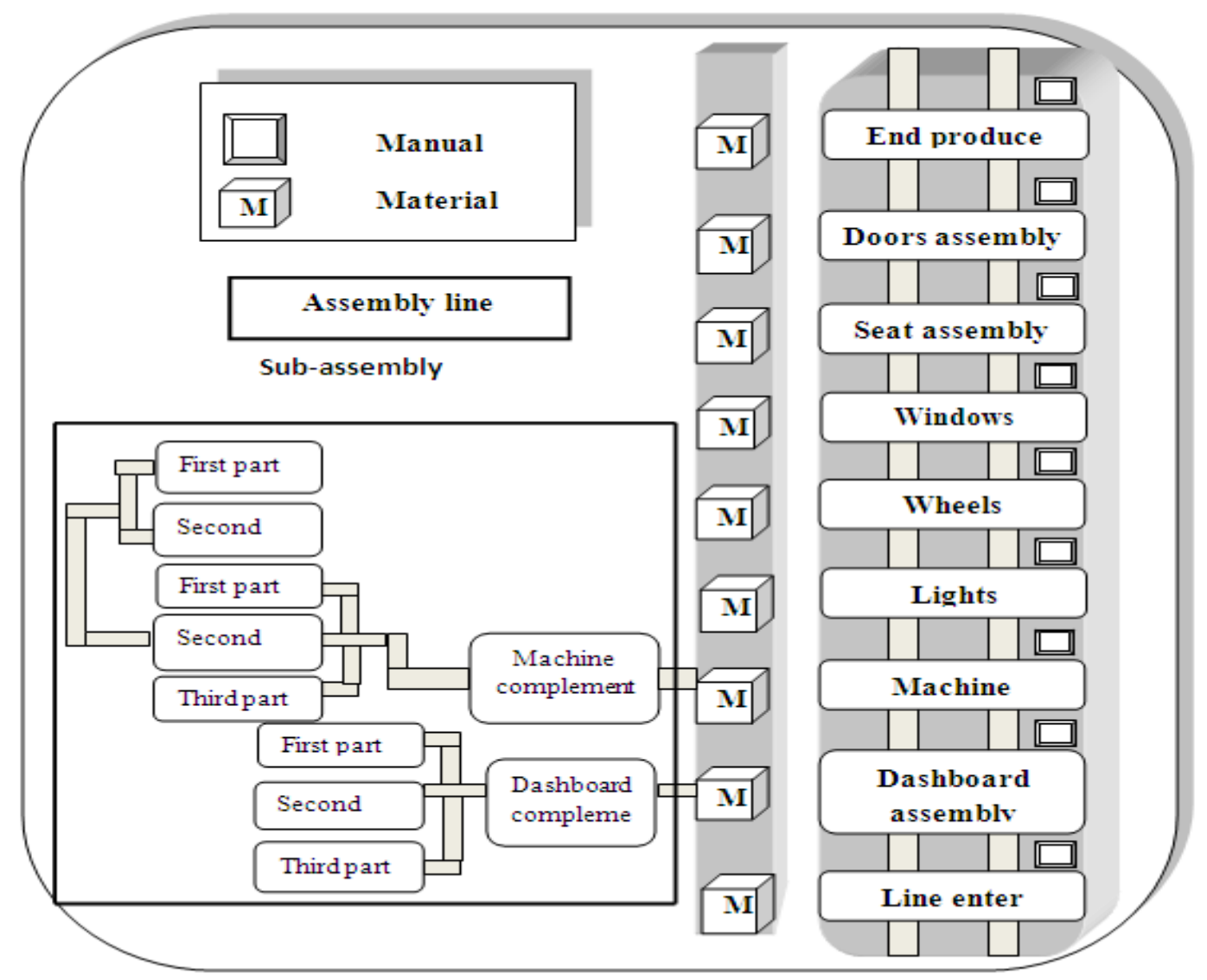

Figure 1. Production line.

This model complemented two goals: The first goal is to increase the production, and the second goal is to lost machine times. They have the subject about how many cars needs and how long time is needed to finish all the orders.

Objectives:

$$
\begin{aligned}
\operatorname{Max} Z & =\sum_{i}^{n} \sum_{j}^{m} C_{i} X_{i j} \\
\operatorname{Min} Z_{2} & =\sum_{i}^{n} \sum_{j}^{m} T_{i j} X_{i j}
\end{aligned}
$$

Subjective:

$$
\begin{aligned}
& \sum_{l}^{n} T_{i} X_{i} \leq D \\
& \sum_{l}^{n} M_{i} X_{i} \leq S
\end{aligned}
$$

where:

$C=$ Amount raw material for product and $X=$ Number of car;

$T=$ Time in machine $i$; 
$D=$ Number of days;

$M=$ Number of the machine;

$S=$ Number of cars should products.

\section{Simulation Process}

The purpose of this paper, we will define simulation as, "the process of designing a computerized model of a system (or process) and conducting experiments with this model for the purpose either of understanding the behavior of the system and/or of evaluating various strategies for the operation of the system”. Thus, we will understand the process of simulation to include both the construction of the model and the analytical use of the model for studying a problem.

The simulation runs in a computer workstation for it costs a lot of computing resources. The process is: Firstly, design variables are assigned with initial values and then product analysis is executed to develop the production plan; Secondly, design variables are extracted from Matlab software.

Figure 2 shows the PL in automobile manufacture. It is containing three shops (body shop, paint shop, and assembly shop). The simulation model applied is to increase the amount of monthly productions in PL (working 8 hours per day). After implementing the MATLAB software the outcome of the model is shown in Table 1 . The total productions monthly are 4,518 cars. Thus, simulation modeling can be considered as an experimental and applied methodology that seeks to accomplish the following:

(1) Describe the behavior of systems;

(2) Construct theories or hypotheses that account for the observed behavior;

(3) Use the model to predict future behavior, i.e., the effects produced by changes in the system or in its method of operation.

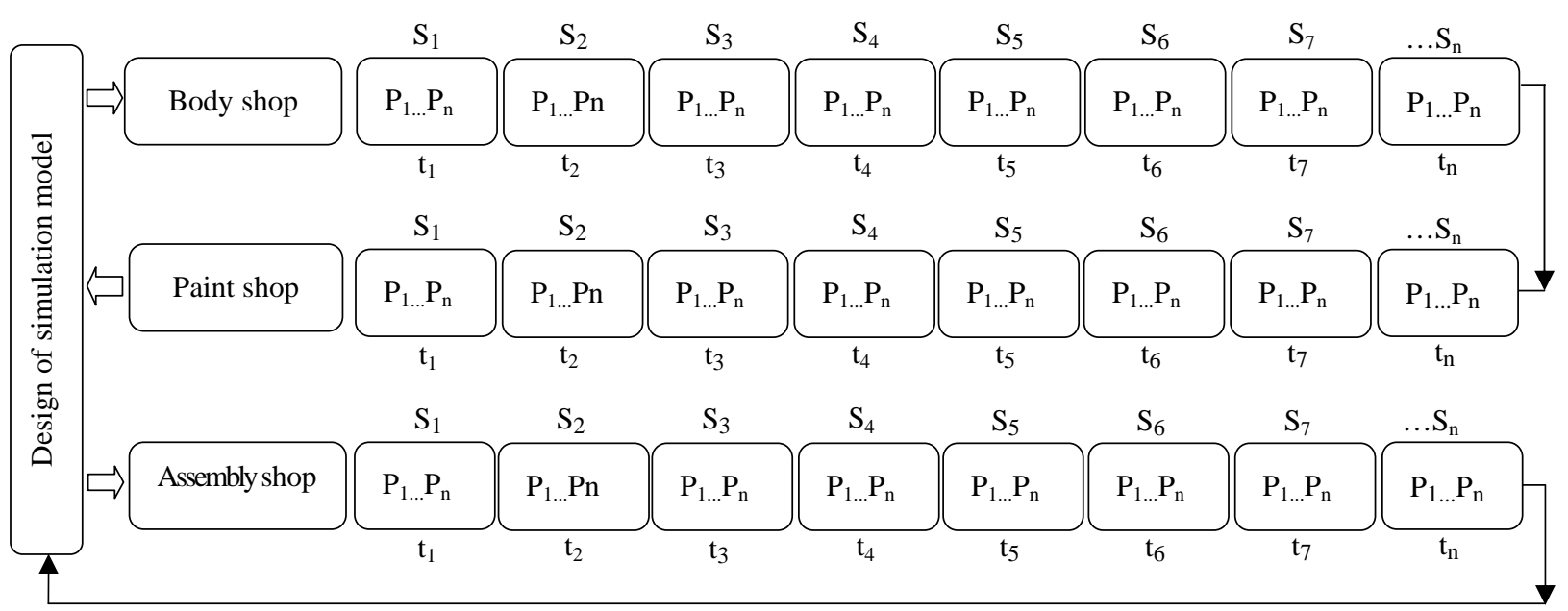

Figure 2. Simulation model.

Table 1

Result

\begin{tabular}{|c|c|c|c|c|c|}
\hline Identifier & Average & Half width & Min & Max & Observations \\
\hline Production time & 4.67 counters & Corr & 4.66 & 4.67 & 4,518 \\
\hline Identifier & & Count & Limit & & \\
\hline Number produced & 4,518 infinite & & & & \\
\hline
\end{tabular}




\section{Result}

The simulation model estimates objective function values when they are needed during the course of optimization, and uses a new rule for accepting a candidate solution that depends on the individual estimated objective function values. The algorithm is shown to converge rapidly on an inventory management problem (base time units: Hours).

- Replication ended at the time: 720 hours;

- Statistics were cleared at the time: 1.0 hours;

- Statistics accumulated for time: 719.0 hours;

- Simulation runs time: 2.37 minutes.

\section{Conclusions}

The main focus of this paper was to develop a production facility that manufactured car based on two models-multi-objectives and simulation model, each depicting a different manufacturing scenario for a large car manufacturing company. The integrated process to get the date can choose produce line optimization that is introduced in detail in this study. Furthermore, the algorithms for multi-objective optimization are emphasized. The model has been integrated with the supply chain simulation developed in another system (MATLAB) using the high level architecture. Production orders and completed order information has been exchanged across simulations. Finally, the company could explore new policies, operating procedures, organizational structures, etc., without disrupting ongoing operations.

\section{References}

Angelisa, E. (2003). The relationships among supply chain characteristics logistics and manufacturing strategies, and performance (Thesis, Ohio University).

Benny, T., \& Raúl, F. (2008). Practical approach to experimentation in a simulation study. Proceedings of the Winter Simulation Conference.

Chen, N., \& Che, H. J. (2009). An integrated method of multi-objective optimization for complex mechanical structure. School of Mechanical Engineering, Southeast University, Nanjing 211189, China.

Dalvi, A., \& Guay, M. (2009). Control and real-time optimization of an automotive hybrid fuel cell power system. Control Engineering Practice, 17, 924-938.

Deogratias, K., \& McLean, C. R. (2007). Generic simulation of automotive assembly for interoperability testing. M.D. 20899, U.S.A, Simulation Conference.

Fritz, J., \& Dolores, H. R. (1997). Simulation of two manufacturing systems for a car manufacturing company (Thesis, College of Engineering and Technology Ohio University).

Javadian, N. J., \& Rezaeian, Y. M. (2007). Multi-objective cellular manufacturing system under machines with different life-cycle using genetic algorithm (Thesis Ohio University).

Jitesh, K. (1998). Maintaining optimum balance between multi skilling and inventory in assembly line operations under dynamic demand. Proceedings of DETC'98, ASME Design Engineering Technical Conferences.

Liu, X. W., Da, Q. L., \& Han, S. L. (1999). Interval number transportation problem model and its solving method based on fuzzy goal programming. Journal of Industrial Engineering and Engineering Management.

Mehrdad, Z. (2004). Manufacturing process effects on fatigue design and optimization of automotive components-An analytical and experimental study. The University of Toledo.

Razmin, B. T., \& Ali, A. J. A. (2009). Multi-object optimization transportation and distribution rice in Malaysia. Proceedings of MUCEET.

Zitzler, E., \& Thiele, L. (1999). Multi-objective evolutionary algorithms: A comparative study and the strength pare to approach. IEEE Trans Vol Compute. 